In the systemic treatment of brain metastases from non-small cell lung cancer (BMF-NSCLC) chemo- and targeted therapy are used. Response rates after platinum-based chemotherapy, range from $23 \%$ to $45 \%$. Development of epidermal growth factor receptor tyrosine kinase inhibitors (EGFR-TKIs): gefitinib or erlotinib, was an improvement in treatment of advanced NSCLC patients. EGFR mutations are present in $10-25 \%$ of NSCLC (mostly adenocarcinoma), and up to $55 \%$ in never-smoking women of East Asian descent. In the non-selected group of patients with BMF-NSCLC, the overall response rates after gefitinib or erlotinib treatment range from $10 \%$ to $38 \%$, and the duration of response ranges from 9 to 13.5 months. In the case of present activating EGFR mutation, the response rate after EGRF-TKIs is greater than $50 \%$, and in selected groups (adenocarcinoma, patients of Asian descent, never-smokers, asymptomatic BMF-NSCLC) even $70 \%$. Gefitinib or erlotinib treatment improves survival of BMF-NSCLC patients with EGFR mutation in comparison to cases without the presence of this mutation. There is no data on the activity of the anti-EML4-ALK agent crizotinib. Bevacizumab, recombinant humanised monoclonal antibody anti-VEGF, in the treatment of advanced non-squamous NSCLC patients is a subject of intense research. Data from a clinical trial enrolling patients with pretreated or occult BMF-NSCLC proved that the addition of bevacizumab to various chemotherapy agents or erlotinib is a safe and efficient treatment, associated with a low incidence of CSN haemorrhages. However, the efficacy and safety of bevacizumab used for therapeutic intent, regarding active brain metastases is unknown.

Key words: breast cancer, brain metastases, systemic therapy, targeted therapy.

Contemp Oncol (Pozn) 2016; 20 (5): 352-357 DOI: $10.5114 /$ wo.2016.64593

\section{Systemic treatment of non-small cell lung cancer brain metastases}

\author{
Ida Cedrych ${ }^{1}$, Maksymilian A. Kruczała ${ }^{1}$, Tomasz Walasek ${ }^{2}$, \\ Jerzy Jakubowicz ${ }^{3}$, Paweł Blecharz ${ }^{4}$, Marian Reinfuss ${ }^{2}$
}

\begin{abstract}
${ }^{1}$ Department of Systemic and Generalised Malignancies, Maria Sklodowska-Curie Memorial Cancer Centre and Institute of Oncology, Krakow Branch, Poland 2Department of Radiotherapy, Maria Sklodowska-Curie Memorial Cancer Centre and Institute of Oncology, Krakow Branch, Poland

${ }^{3}$ Department of Oncology, Maria Sklodowska-Curie Memorial Cancer Centre and Institute of Oncology, Krakow Branch, Poland

${ }^{4}$ Department of Gynecologic Oncology, Maria Sklodowska-Curie Memorial Cancer Centre and Institute of Oncology, Krakow Branch, Poland
\end{abstract}

\section{Introduction}

Between $25 \%$ and $30 \%$ of non-small cell lung cancer (NSCLC) patients will develop metastatic disease in the brain (brain metastases from non-small cell lung cancer - BMF-NSCLC). Frequently they are the first site of recurrence in early-stage NSCLC patients treated with definitive therapies [1-5]. The prognosis is poor for untreated patients with BMF-NSCLC, with median overall survival (OS) $1-2$ months $[1,4,5]$. The combination of neurosurgery with stereotactic radiosurgery (SRS) and/or whole-brain radiotherapy (WBRT) can increase the OS up to 3-6 months, and in selected cases over 12 months [1, 4, 6-10].

Currently, the role of systemic treatment of BMF-NSCLC patients is being widely discussed $[3,4,10]$. Historically, chemotherapy was considered as a poorly effective method of treatment, mainly because of predicted difficulties in penetrating the blood-brain-barrier (BBB). For a long period of time, patients with BMF-NSCLC were excluded from controlled clinical trials for chemotherapy of $\operatorname{NSCLC}[1,3,4,11,12]$. Nowadays it seems that even if most of the drugs cannot penetrate normal $\mathrm{BBB}$, the integrity of the $\mathrm{BBB}$ is significantly altered, e.g. in BMF-NSCLC patients, which can be proved by oedema and increased contrast uptake around the metastatic site [12]. The significant amount of information indicates the possibility of efficient palliative systemic treatment of chosen patients with BMF-NSCLC $[2,3,10,11,13]$. The role of targeted therapies, besides chemotherapy, is significantly increasing $[1,4,10,13,14]$.

The purpose of this work is to review, relying on the literature, the actual knowledge on the methods and results of systemic treatment of brain metastases from non-small cell lung cancer.

\section{Chemotherapy}

Recent phase II trials indicate efficacy, however limited, of platinum-based chemotherapy of BMF-NSCLC patients [15-20], which is presented in Table 1.

Phase II trials demonstrating efficacy of first-line BMF-NSCLC chemotherapy.

As outlined in Table 1, the response rates after platinum-based chemotherapy range from 23\% to 45\%; Chaubet-Houdu and Basse report 23-50\%. Literature indicates that temozolomide (TMZ) combined with radiotherapy, in BMF-NSCLC, has a slight influence on survival, but it might increase the toxicity of the treatment [2,11, 21-23].

\section{Tyrosine kinase inhibitors}

Development of epidermal growth factor receptor tyrosine kinase inhibitors (EGFR-TKIS): gefitinib or erlotinib, has clearly improved the treatment of 
Table 1. Efficacy of platinum-based chemotherapy of BMF-NSCLC patients in phase II trials

\begin{tabular}{|c|c|c|c|c|}
\hline $\begin{array}{l}\text { Authors, publication date, } \\
\text { reference no. }\end{array}$ & Chemotherapy & Number of patients & $\begin{array}{l}\text { Overall response rate } \\
\text { (\%) }\end{array}$ & $\begin{array}{c}\text { Median overall survival } \\
\text { (months) }\end{array}$ \\
\hline Cotto et al. 1996 [15] & $\begin{array}{l}\text { cisplatin + } \\
\text { fotemustine }\end{array}$ & 31 & 23 & 4 \\
\hline Minotti et al. 1998 [16] & $\begin{array}{l}\text { cisplatin + } \\
\text { teniposide }\end{array}$ & 23 & 35 & 5 \\
\hline Franciosi et al. 1999 [17] & $\begin{array}{l}\text { cisplatin + } \\
\text { etoposide }\end{array}$ & 43 & 30 & 8 \\
\hline Bernardo et al. 2002 [18] & $\begin{array}{l}\text { carboplatin, } \\
\text { navelbine, } \\
\text { gemcitabine }\end{array}$ & 22 & 45 & 8 \\
\hline Cortes et al. 2003 [19] & $\begin{array}{l}\text { cisplatin + } \\
\text { taxol }\end{array}$ & 26 & 38 & 5 \\
\hline Barlesi et al. 2011 [20] & $\begin{array}{l}\text { cisplatin }+ \\
\text { pemetrexed }\end{array}$ & 43 & 42 & 7 \\
\hline
\end{tabular}

advanced NSCLC patients [3, 4, 9, 10, 13, 24-45]. EGFR mutations are present in 10-25\% of NSCLC, with the highest prevalence found in never-smoking women of East Asian descent (up to 55\%) [13, 24]. Paez et al. and Pao et al. found EGFR mutations to be present in $63 \%$ and $50 \%$ of BMFNSCLC patients, respectively, which suggests increased risk of developing brain metastases among patients with these mutations $[25,26]$.

In a non-selected group of patients with BMF-NSCLC the overall response rates after gefitinib range from $10 \%$ to $38 \%$, and the duration of response ranges from 9 to 13.5 months [27-30]; erlotinib has similar efficacy [31-35]. It seems that erlotinib achieves higher central nervous system (CNS) concentration in comparison to gefitinib [10, 13]. Gefitinib and erlotinib are both approved as first-line treatment, palliative treatment (second- and third-line), and in combination with radiotherapy (WBRT \pm SRS), their efficacy was presented in case reports, case series, and nonrandomised phase II trials [2, 27, 31, 38, 40, 42, 45].

Two phase II trials evaluated the efficacy of TKI in the first-line setting on patients with BMF-NSCLC $[38,40]$. Both trials did not include data for EGFR mutations, whereas the studies included never-smokers. Lee et al. [40] reported 10 patients; seven demonstrated an objective response to gefitinib, one had a stable disease, and two had a progressive disease after a median 48-week follow-up period. Kim et al. [38] presented a group of 23 patients with synchronous BMF-NSCLC with a response rate to gefitinib or erlotinib of $69 \%$ and median overall survival of 18.8 months. Heon et al. analysed a group of 155 patients with BMF-NSCLC screened for EGFR mutations [41]. The rate of CNS progression was lower among EGRF-mutant patients treated with gefitinib or erlotinib compared with upfront chemotherapy (patients without EGFR mutation) - 33\% vs. $48 \%$, respectively, at a median follow-up of 25 months.

Two phase II trials assessed the role of gefitinib in the palliative setting in non-selected patients with BMF-NSCLC [27, 31]. Ceresoli et al. [27] reported 41 patients with a 10\% response rate and median overall survival of five months, Wu et al. [31] reported 40 patients (adenocarcinoma, never-smokers) with a $32 \%$ response rate and median overall survival of 15 months. Pesce et al. [45] in a randomised study comparing WBRT + gefitinib vs. WBRT + TMZ, failed to show an advantage of gefitinib in a non-selected group of patients with BMF-NSCLC; OS 6.3 months in the gefitinib arm and 4.9 months in the TMZ arm, the difference was statistically irrelevant.

A phase III clinical trial conducted by Sperduto et al. [2] showed that TMZ or erlotinib combined with WBRT + SRS in a non-selected group of patients with 1-3 BMP-NSCLC did not improve the OS; however, it increased the toxicity of the treatment.

Welsh et al. study [42] evaluated the efficacy of erlotinib in combination with WBRT in 40 patients with BMF-NSCLC. Patients negative for EGFR mutations had a median overall survival of 9.3 months, whereas patients positive for EGFR mutations had 19.1 months. It is also undoubted that either gefitinib or erlotinib can be safely combined with WBRT $[43,44]$.

Some authors suggest that in selected groups of patients with BMF-NSCLC, commencing treatment with gefitinib or erlotinib, with delayed WBRT, is acceptable. It relates to women with adenocarcinoma, never-smokers, and patients positive for EGFR mutations. Iuchi et al. presented good efficacy of gefitinib alone (without radiotherapy) in patients with adenocarcinoma BMF-NSCLC, positive for EGFR mutation - median overall survival 21.9 months in a group of 41 patients [3]. The phase II APRAGE trial, comparing WBRT + gefitinib with gefitinib alone in BMF-NSCLC patients, is ongoing $[3,12]$.

In conclusion, TKI (gefitinib, erlotinib) overall response rate depends essentially on the presence of EGFR gene activating mutation $[10,12,13,36,37]$; if mutation is present, ORR reaches more than 50\% [12]. In non-selected groups of patients (adenocarcinoma, Asian descents, never-smokers, asymptomatic BMF-NSCLC) after TKIs therapy, it is possible to reach $70 \%$ ORR $[13,38]$. TKIs improve survival of BMF-NSCLC patients with EGFR mutations in comparison to patients without these mutations $[10,12,13,39]$. 


\section{Crizotinib}

In approximately $3-5 \%$ of patients with NSCLC, an ALK (anaplastic lymphoma kinase) rearrangement occurs. It results in forming an EML4-ALK fusion gene; it relates to mostly young, male, never-smokers, with adenocarcinoma [10, 12-14, 46]. In this group, administration of crizotinib, an anti-EML4-ALK (echinoderm microtubule-associated protein-like 4-anaplastic lymphoma kinase) drug, is reasonable and effective [10, 14, 46-48].

Kwak et al. [46] reported a 57\% response rate, and a randomised phase III trial presented by Shaw et al. [47] indicated statistically relevant improvement of progression-free survival of subjects treated with crizotinib, compared to patients treated with a second-line chemotherapy (pemetrexed or docetaxel). Unfortunately, crizotinib has a poor BBB penetration, so its efficacy in BMF-NSCLC patients is doubtful $[10,13,14,46,49-51]$. The available literature provides poor corresponding data [12, 49, 50, 52]. Chun et al. presented a case of BMF-NSCLC progression during crizotinib treatment, despite regression of the disease outside CNS [49]. Weickhardt et al. reported on crizotinib in 38 ALK (+) patients; 28 demonstrated progressive disease, and in $46 \%$ the first site of recurrence was BMF-NSCLC. Among patients with isolated recurrence in BMF-NSCLC, treated with radiotherapy (WBRT or SRS) followed by crizotinib, progression-free survival of 7.1 months was obtained [52].

Single cases of BMF-NSCLC responsive to crizotinib were reported by Kaneda et al. [53] and Kinoshita et al. [48]. Kinoshita suggest that administering ionising radiotherapy before crizotinib treatment may play an important role in both cases $[43,48]$. In 2006 Yuan et al. indicated in a murine model that CNS radiotherapy increases penetrability of the BBB [54]. Mehra et al., in a phase I trial, demonstrated responses in BMF-NSCLC patients treated with one of the new generation of ALK inhibitors - LDK 378 [55].

\section{Bevacizumab}

Bevacizumab is a humanised monoclonal antibody that binds selectively to VEGF - vascular endothelial growth factor. Blocking the VEGF protein should result in impairment of tumour blood vessel growth. Eventually, cancer cells should not develop their own blood supply, causing a lack of oxygen and nutrients, helping to slow down the growth of the tumour. Treatment of advanced NSCLC with bevacizumab remains controversial $[1,12,13]$. Results of two randomised phase III trials, ECOG 4599 and AVAil, reported that bevacizumab combined with chemotherapy improved the response rate and progression-free survival compared to chemotherapy alone in NSCLC. ECOG 4599 also reported a significantly longer OS (12.3 vs. 10.3 months) [56-58]. However, the patient population was restricted to non-squamous histology. Hypertension, massive haemoptysis, disorders in blood coagulation, and BMF-NSCLC were also qualified as exclusion criteria. The restriction of the patient population to non-squamous histology was based on the research of Johnson et al., which indicated the occurrence of life-threatening haemoptysis in this group (4/13 patients) [59]. Exclusion of BMF-NSCLC patients was based on the current opinion that bevacizumab significantly increase the risk of intracranial bleeding in this group $[1,12,13]$. In both trials, the incidence of CNS haemorrhages among patients receiving bevacizumab was similar to the incidence of those reported in patients who did not receive bevacizumab. Based on the results of these trials, bevacizumab is currently licensed for use ase first-line therapy in combination with chemotherapy (carboplatin + paclitaxel) in the USA, or in addition to platinum-based chemotherapy in Europe in patients with advanced non-squamous NSCLC [1]. However, it does not mean it is commonly used; this is because of absent or poor benefit compared to chemotherapy alone, with a slightly increased toxicity [60].

It is obvious that there are no reasons to exclude patients with brain metastases from clinical trials on antiangiogenic agents, as took place in the recent past $[1,13,56]$. Despite antiangiogenic therapy, patients with or without brain metastases have similar risk of intracranial bleeding (90.8-3.3\%) [60-64].

Several retro- and prospective clinical trials conducted in the past few years indicate that the combination of bevacizumab with chemotherapy or erlotinib is safe in the treatment of BMF-NSCLC, with a slight risk of intracranial bleeding [60, 62-68].

A prospective phase IV study ARIES evaluated the safety and efficacy of the first-line setting in patients with non-squamous NSCLC treated with bevacizumab combined with chemotherapy. A total of 150 patients with BMF-NSCLC were enrolled, median PFS and OS were 6.0 and 11.7 months, respectively, and no grade 3 to 5 CNS haemorrhage occurred [65].

The phase II study PASSPORT enrolled 115 NSCLC patients with previously treated BMF-NSCLC with WBRT and/ or surgery. Patients received as a first-line bevacizumab, with platinum-based doublet chemotherapy or erlotinib, and as a second-line, bevacizumab with single-agent chemotherapy or erlotinib; no grades 1 to 5 CNS haemorrhage, among patients who received bevacizumab-based therapy were reported [62].

The phase III ATLAS study was designed to evaluate the combination of bevacizumab/erlotinib versus bevacizumab/placebo as maintenance therapy after four cycles of induction platinum-containing chemotherapy plus bevacizumab as first-line treatment in advanced NSCLC patients. Among 25 evaluable patients with a history of CNS metastases pretreated with WBRT and/or neurosurgery, one grade 2 CNS bleeding was observed in a patient on post-progression therapy after 14 cycles of bevacizumab $[66,67]$.

The SAiL study assessed the safety and efficacy of the addition of bevacizumab to first-line chemotherapy. This study proved that bevacizumab-based therapy resulted in median OS of 14.6 months, with a median time to disease progression of 7.8 months. Efficacy was generally similar across chemotherapy regimens. The specific safety of bevacizumab was assessed in patients who either developed BMF-NSCLC during treatment or had occult BMF-NSCLC at 
study entry. Of the 281 patients evaluated, five (2\%) had CNS bleeding [60].

The phase III BeTaLung study evaluated the addition of bevacizumab to erlotinib for the second-line treatment of advanced NSCLC patients. A total of 636 patients were randomised to receive bevacizumab in combination with either erlotinib or erlotinib alone. The addition of bevacizumab to erlotinib increased PFS compared to erlotinib alone (3.4 vs. 1.7 months, respectively). This trial included patients with BMF-NSCLC, previously treated with WBRT and neurosurgery or WBRT + SRS. Among 68 BMF-NSCLC patients, 37 received erlotinib + bevacizumab and 31 erlotinib alone. No CNS haemorrhage or grade $>3$ bleeding was reported in either arm [68].

Besse et al. presented an analysis including more than 12,000 advanced/metastatic breast cancer, NSCLC, renal, and colorectal cancer patients, with previously treated CNS metastases, from 13 phase II/II randomised controlled trials, two open-label, single-arm safety studies, and two prospective studies. The rate of cerebral haemorrhage in the bevacizumab-treated group was 3.3\%, compared to $1 \%$ in the group not treated with bevacizumab. This study suggests that the administration of bevacizumab should no longer be contraindicated based solely on the presence of CNS metastases [63].

Several clinical trials have been launched to determine the safety and efficacy of various other antiangiogenic agents in the treatment of new or progressive brain metastases from solid tumours: sunitinib, cediranib, and vatalanib [1].

In conclusion:

1. Chemotherapy is generally effective in BMF-NSCLC, and platinum-based provides response rates ranging from $23 \%$ to $45 \%$

2. Epidermal growth factor receptor tyrosine kinase inhibitors (EGFR - TKIS) - gefitinib and erlotinib - have a definite activity in BMF-NSCLC with activating EGFR mutation, or in selected groups of patients (woman of east Asian descent, never-smokers, those with adenocarcinoma); the response rate ranges from $38 \%$ to $70 \%$. Both EGFR-TKIs have been investigated in first-line, palliative, and in combination with radiotherapy. Patients with BMF-NSCLC-EGFR-mutant have improved overall survival compared with EGFR wild-type tumours, when receiving an EGFR inhibitor.

3. There is no data on the activity of the agent ani-EML4ALK-crizotinib in patients with BMF-NSCLC. Crizotinib has a poor penetration of BBB.

4. Data from a clinical trial enrolling patients with pretreated or occult BMF-NSCLC showed that the addition of bevacizumab to various chemotherapy agents or erlotinib is a safe and efficient treatment, associated with a low incidence of CNS haemorrhage. However, bevacizumab should be used with caution in patients with active BMF-NSCLC.

The authors declare no conflict of interest.

\section{References}

1. Schettino C, Bareschino MA, Rossi A, Maione P, Sacco PC, Colantouni G, Rossi E, Gdidelli C. Targeting angiogenesis for treatment of NSCLC brain metastases. Curr Cancer Drugs Targets 2012; 12: 289-99.

2. Sperduto PW, Wang M, Robis HI, et al. A phase 3 trial of whole brain radiation therapy and stereotactic radiosurgery alone versus WBRT and SRS with temozolomide or erlotinib for non-small cell lung cancer and 1 to 3 brain metastases: Radiation Therapy Oncology Group 0320. Int J Radiat Oncol Biol Phys 2013; 85: 1312-8.

3. Iuchi T, Shingyoji M, Sakaida T, et al. Phase II trial of gefitinib alone without radiation therapy for Japanese patients with brain metastases from EGFR-mutant lung adenocarcinoma. Lung Cancer 2013; 82: 282-7.

4. Villalva C, Duranton-Tanneur V, Guilloteau K, et al. EGRF, KRAS, BRAF and HER-2 molecular status in brain metastases from 77 NSCLC patients. Cancer Med 2013; 2: 296-304.

5. Barlesi F, Khobta N, Tablet A, et al. Strategies de prise en charge des metastases cerebrales des maladies atteints de cancers bronchiques primitives. Bull Cancer 2013; 100: 303-8.

6. Bradley KA, Mehta MP. Management of brain metastases. Semin Oncol 2004; 31: 693-701.

7. Andrews DW, Scott CB, Sperduto PW, et al. Whole brain radiation therapy with or without stereotactic radiosurgery boost for patients with one to three brain metastases: phase III results for the RTOG 9508 randomised trial. Lancet 2004; 363: 1665-72.

8. Sundstrom JT, Minn H, Lertola, Nordman E. Prognosis of patients treated for intracranial metastases with whole-brain irradiation. Ann Med 1998; 30: 296-9.

9. Bailon O, Kallel A, Chouahnia K, Billot S, Ferrari D, Carpentier AF. Management of brain metastases from non-small cell lung carcinoma. Revue Neurol 2011; 16: 579-91.

10. Renfrow JJ, Lesser GJ. Molecular subtyping of brain metastases and implications for therapy. Curr Treat Options Oncol 2013; 14: 514-27.

11. Hassler MR, Pfeifer W, Knocke-Abulesz TH, Geissler K, Altorjai G, Dieckmann K, Marosi C. Temozolomide added to whole brain radiotherapy in patients with multiple brain metastases of nonsmall-cell lung cancer: a multicentric Austrian phase II study. Wien Klin Wochenschr 2013; 125: 481-6.

12. Chaubet-Houdu M, Besse B. Metastases cerebrales des cancers bronchiques non a petites cellules: traitement systemique Bull Cancer 2013; 100: 95-8.

13. Soffietti R, Trevisan E, Ruda R. Targeted therapy in brain metastasis. Curr Opin Oncol 2012; 24: 679-86.

14. Levitt MR, Levit R, Silbergelt DL. Controversies in the management of brain metastases. Surg Neurol Int 2013; 4: S231-5.

15. Cotto C, Berille J, Souquet PJ, et al. A phase II trial of fotemustine and cisplatin in central nervous system metastases from nonsmall cell lung cancer. Eur J Cancer 1996; 32A: 69-71.

16. Minotti V, Crino L, Meacci ML, Corgana E, Darwish S, Palladino MA, Betti M, Tonato $M$. Chemotherapy with cisplatin and teniposide for cerebral metastases in non-small cell lung cancer. Lung Cancer 1998; 20: 93-8.

17. Franciosi V, Cocconi G, Michiara M, et al. Front-line chemotherapy with cisplatin and etoposide for patients with brain metastases from breast carcinoma, nonsmall cell lung carcinoma, or malignant melanoma: a prospective study. Cancer 1999; 85: 1599-605.

18. Bernardo G, Cuzzoni O, Strada MR, Bernardo A, Brunetti G, Jedrychowska I, Pozzi U, Palumbo G. First-line chemotherapy with vinorelbine, gemcitabine and carboplatin in the treatment of brain metastases from non-small cell lung cancer: a phase II study. Cancer Invest 2002; 20: 293-302.

19. Cortes J, Rodriguez J, Aramendia JM, et al. Front-line paclitaxel/cisplatin based chemotherapy in brain metastases from non-small cell lung cancer. Oncology 2003; 64: 28-35.

20. Barlesi F, Gervais R, Lena H, et al. Pemetrexed and cisplatin as first-line chemotherapy for advanced non-small cell lung cancer (NSCLC) with asymptomatic inoperable brain metastases: a multicenter phase II trial (GFPC 07-01). Ann Oncol 2011; 22: 2466-70. 
21. Giorgio CG, Giuffrida D, Pappalardo A, et al. Oral temozolomide in heavily pre-treated brain metastases from non-small cell lung cancer: phase II study. Lung Cancer 2005; 50: 247-54.

22. Dziadziuszko R, Ardizzoni A, Postmus PE, Smit EF, Price A, Debruyne C, Legrand C, Giaccone G. Temozolomise in patients with advanced non-small cell lung cancer with and without brain metastases. A phase II study of the EORTC Lung Cancer Group (08965). Eur J Cancer 2003; 39: 1271-6.

23. Chua D, Krzakowski M, Chouaid C, Wallota MG, Martinem J, Gottfried M, Curran W, Throvalas N. Whole brain radiation therapy plus concominant temozolomide for the treatment of brain metastases from non-small cell lung cancer: a randomized, open-label phase II study. Clinical Lung Cancer 2010; 11: 176-81.

24. Chen AM, Jahan TM, Jablons DM, Garcia J, Larson DA. Risk of cerebral metastases and neurological death after pathological complete response to nneoadjuvant therapy for locally advanced nonsmall-cell lung cancer: clinical implications for the subsequent management of the brain. Cancer 2007; 109: 1668-75.

25. Paez JG, Janne PA, Lee JC, et al. EGFR mutations in lung cancer: correlation with clinical response to gefitinib therapy. Science 2004 304: 1497-500.

26. Pao W, Miller V, Zakowski M, et al. EGF receptor gene mutations are common in lung cancers from "never smokers" and are associated with sensitivity of tumors to gefitinib and erlotinib. Proc Natl Acad Sci U S A 2004; 101: 13306-11.

27. Ceresoli GL, Capuzzo F, Gregorc V, Bartolini S, Crino L, Villa E. Gefitinib in patients with brain metastases from non-small cell lung cancer: a prospective trial. Ann Oncol 2004; 15: 1042-7.

28. Hotta K, Kiura K, Ueoka H, et al. Effect of gefitinib ("Iressa", ZD1839) on brain metastases in patients with advanced nonsmall cell lung cancer. Lung Cancer 2004; 46: 255-61.

29. Namba Y, Kijima T, Yokota S, et al. gefitinib in patients with brain metastases from non-small cell lung cancer: review of 15 clinical cases. Clin Lung Cancer 2004; 6: 123-8.

30. Chiu CH, Tsai CM, Chen YM, Chiang SC, Liou JL, Perng RP. Gefitinib is active in patients with brain metastases from non-small cell lung cancer and response is related to skin toxicity. Lung Cancer 2005; 47: 129-38.

31. Wu C, Li YL, Wang ZM, Wei Z. Gefitinib as palliative therapy for lung adenocarcinoma metastasis to the brain. Lung Cancer 2007; 57: 359-64.

32. Fekrazad MH, Ravindranathan M, Jones DV Jr. Response of intracranial metastases to erlotinib therapy. J Clin Oncol 2007; 25: 5024-6.

33. Popat S, Hughes S, Papadopoulos P, et al. Recurrent responses to non-small cell lung cancer brain metastases with erlotinib. Lung Cancer 2007; 56: 135-7.

34. Altavilla G, Arrigo C, Santarpia MC, Galletti G, Picone G, Marabello G, Tomasello C, Pitini VV. Erlotinib therapy in a patient with nonsmall cell lung cancer and brain metastases. J Neurooncol 2008; 90: 31-3.

35. Bai H, Han B. The effectiveness of erlotinib against brain metastases in non-small cell lung cancer patients. Am J Clin Oncol 2013; 36: 110-5.

36. Porta R, Sanchez-Torres JM, Paz-Ares L, et al. Brain metastases from lung cancer responding to erlotinib: the importance of EGFR mutation. Eur Respir J 2011; 37: 624-31.

37. Shimato S, Mitsudomi T, Kosaka T, et al. EGFR mutations in patients with brain metastases from lung cancer: association with the efficacy of gefitinib. Neuro Oncol 2006; 8: 137-44.

38. Kim JE, Lee DH, Choi Y, Yoon DH, Kim SW, Suh C, Lee JS. Epidermal growth factor receptor tyrosine kinase inhibitors as first-line therapy for never-smokers with adenocarcinoma of the lung having asymptomatic synchronous brain metastases. Lung Cancer 2009; 65: 351-4

39. Eichler AF, Kahle KT, Wang DL, Joshi VA, Willers H, Engelman JA, Lynch TJ, Sequist LV. EGFR mutation status and survival after diagnosis of brain metastases in nonsmall cell lung cancer. Neuro Oncol 2010; 12: 1193-9.

40. Lee DH, Han JY, Lee HG, et al. Gefitinib as first-line therapy of advanced or metastatic adenocarcinoma of the lung in never-smokers. Clin Cancer Res 2005; 11: 3032-7.
41. Heon S, Yeap BY, Lindeman NI, et al. The impoact of initial gefitinib or erlotinib versus chemotherapy on central nervous system pro gression in advanced non-small cell lung cancer with EGFR mutations. Clin Cancer Res 2012; 18: 4406-14.

42. Welsh JW, Komaki R, Amini A, et al. Phase II trial of erlotinib plus concurrent whole-brain radiation therapy for patients with brain metastases from non-small cell lung cancer. J Clin Oncol 2013; 31: 895-902

43. Lind JS, Lagerwaard FJ, Smit EF, Senan S. Phase I study of concurrent whole brain radiotherapy and erlotinib for multiple brain metastases from non-small cell lung cancer. Int J Radiat Oncol Biol Phys 2009; 74: 1391-6.

44. Ma S, Xu Y, Deng Q, Yu X. Treatment of brain metastasis from nonsmall cell lung cancer with whole brain radiotherapy and Gefitinib in a Chinese population. Lung Cancer 2009; 65: 198-203.

45. Pesce GA, Klingbiel D, Ribi K, et al. Outcome, quality of live and cognitive function of patients with brain metastases from nonsmall cell lung cancer treated with whole brain radiotherapy combined with gefitinib or temozolomide. A randomized phase II trial of the Swiss Group for Clinical Cancer Research (SAKK 70/03). Eur J Cancer 2012; 48: 377-84.

46. Kwak EL, Bang YJ, Camidge DR, et al. Anaplastic lymphoma kinase inhibition in non-small cell lung cancer. N Eng J Med 2010; 363: 1693-703.

47. Shaw AT, Kim DW, Nakagawa K, et al. Phase III study of crizotinib versus pemetrexed or docetaxel chemotherapy in patients with advanced ALK positive non-small cell lung cancer (NSCLC) (PROFILE 1007); Abstract LBA1 presented at the Annual Congress of the European Society for Medical Oncology; Vienna, Austria. September 28-October 2, 2012.

48. Kinoshita Y, Koga Y, Sakamoto A, Hidaka K. Long-lasting response to crizotinib in brain metastases due to EML4-ALK-rearranged non-small cell lung cancer. BMJ Case Rep 2013. doi: 10.1136/bcr2013-200867.

49. Chun SG, Choe KS, lyengar P, Yordy JS, Timmerman RD. Isolated central nervous system progression on Crizotinib: an Achilles heel of non-small cell lung cancer with EML4-ALK translocation? Cancer Biol Ther 2012; 13: 1376-83.

50. Costa DB, Kobayashi S, Pandya SS, Yeo WL, Shen Z, Tan W, Wilner KD. CSF concentration of the anaplastic lymphoma kinase inhibitor crizotinib. J Clin Oncol 2011; 29: e443-5.

51. Falk AT, Poundex M, Otto J, Ghalloussi H, Barriere J. Adenocarcinoma of the lung with military brain and pulmonary metastases with echinoderm microtubule-associated protein like 4-anaplastic lymphoma kinase translocation treated with crizotinib: a case report. Lung Cancer 2012; 78: 282-4.

52. Weickhardt AJ, Scheier B, Burke JM, et al. Continuation of EGFR/ ALK inhibitor after local therapy of oligoprogressive disease in EGFR mutant (Mt) and ALK+ non-small cell lung cancer (NSCLC). J Clin Oncol 2012; 30 (suppl) abstr 7526.

53. Kaneda H, Okamoto I, Nakagawa K. Rapid response of Brain Metastasis to Crizotinib in a Patient with ALK Rearrangement-Positive Non-Small-Cell Lung Cancer. J Thorac Oncol 2013; 8: e32-3.

54. Yuan H, Gaber MW, Boyd K, Wilson CM, Kiani MF, Merchant TE. Effects of fractionated radiation on the brain vasculature in a murine model: blood-brain barrier permeability, astrocyte proliferation and ultrastructural changes. Int J Radiat Oncol Biol Phys 2006; 66: 860-6.

55. Mehra R, Cambridge DR, Sharma S, et al. First-in human phase I study of the ALK inhibitor LDK378 in advanced solid tumors (abstract) in: Journal of Clinical Oncology ASCO Annual Meeting Proceedings: 2012 May 20; Chicago, Illinois (IL): ASCO; 2012; 30 (15s): 3007.

56. Sandler A, Gray R, Perry MC, Brahmer J, Schiller JH, Dowlati A, Lilenbaum R, Johnson DH. Paclitaxel-carboplatin alone or with bevacizumab for non-small cell lung cancer. N Eng J Med 2006; 355: 2542-50

57. Reck M, von Pawel J, Zatloukal P, et al. Overall survival with cisplatin-gemcitabine and bevacizumab or placebo as first-line therapy for nonsquamous non-small cell lung cancer: results from a randomized phase III trial (AVAiL). Ann Oncol 2010; 21: 1804-9. 
58. Reck M, von Pawel J, Zatloukal P, et al. Phase III trial of cisplatin plus gemcitabine with either placebo or bevacizumab as first-line therapy for non-small cell lung cancer: AVAiL. J Clin Oncol 2009; 27: $1227-34$

59. Johnson DH, Fehrenbacher L, Novotny WF, et al. Randomized phase II trial comparing bevacizumab plus carboplatin and packlitaxel with carboplatin and packlitaxel alone in previously untreated locally or advanced non-small cell lung cancer. I Clin Oncol 2004; 22: 2184-91.

60. Crino L, Danis E, Garrido P, et al. Safety and efficace of first-line bevacizumab-based therapy in advanced non-small cell lung cancer (SAiL, MO 19390): a phase 4 study. Lancet Oncol 2010; 11: 733-40.

61. Gore ME, Szczylik C, Porta C, et al. Safety and efficacy of sunitinib for metastatic renal-cell carcinoma: an expanded-access trial. Lancet Oncol 2009; 10: 757-63.

62. Socinski MA, Langer CJ, Huang JE, Kolb MM, Compton P, Wang L, Akerley W. Safety of bevacizumab in patients with non-small cell lung cancer and brain metastases. J Clin Oncol 2009; 27: 5255-61.

63. Besse B, Lasserre SF, Compton P, Huang J, Augustus S, Rohr UP. Bevacizumab safety in patients with central nervous system metastases. Clin Cancer Res 2010; 16: 269-78.

64. De Braganca KC, Janjigian YY, Azzoli CG, et al. Efficacy and safety of bevacizumab in active brain metastases from non-small cell lung cancer. J Neurooncol 2010; 100: 443-7.

65. Wozniak AJ, Garst J, Jahanzeb M, et al. Clinical outcomes (CO) for special populations of patients (pts) with advanced non-small cell lung cancer (NSCLC): Results from ARIES, a bevacizumab (BV) observational cohort study (OCS). J Clin Oncol 2010; 28:15s (suppl) abstr. 7618 .

66. Miller VA, O'Connor P, Soh C, Kabbinavar F. A randomized, double-blind, placebo-controlled, phase IIIb trial (ATLAS) comparing bevacizumab (B) therapy with or without erlotinib (E) after completion of chemotherapy with B for first-line treatment of locally advanced, recurrent, or metastatic non-small cell lung cancer (NSCLC). J Clin Oncol 2009; 27: 18s (suppl) abstr. LBA 8002.

67. Kabbinavar FF, Miller VA, Johnson BE, O’Connor PG, Soh C. Overall survival (OS) in ATLAS, a phase IIIb trial comparing bevacizum$a b(B)$ therapy with or without erlotinib (E) after completion of chemotherapy (chemo) with B for first-line treatment of locally advanced, recurrent, or metastatic non-small cell lung cancer (NSCLC). J Clin Oncol 2010; 28:15s (suppl) abstr. 7526.

68. Herbst RS, Ansari R, Bustin F, et al. Efficacy of bevacizumab plus erlotinib versus erlotinib alone in advanced non-small cell lung cancer after failure of standard first-line chemotherapy (BeTa): a double-blind, placebo-controlled, phase III trial. Lancet 2011; 377: 1846-54.

\section{Address for correspondence}

\section{Maksymilian A. Kruczała}

Department of Systemic and Generalised Malignancies

Maria Sklodowska-Curie Memorial Cancer Centre

and Institute of Oncology

Garncarska 11

31-115 Krakow Branch, Poland

e-mail:m_kruczala@onet.pl

Submitted: 2.04 .2014

Accepted: 27.07.2015 